\title{
Exploring the pros and cons of mechanistic case diagrams for problem-based learning
}

\author{
Minjeong Kim ${ }^{1}$ and Bong Jin Kang ${ }^{2}$ \\ ${ }^{1}$ Department of Teaching Education, Dankook University College of Education, Yongin, and ${ }^{2}$ Department of Anesthesia \\ and Pain Medicine, Dankook University College of Medicine, Cheonan, Korea
}

Purpose: Mechanistic case diagram (MCD) was recommended for increasing the depth of understanding of disease, but with few articles on its specific methods. We address the experience of making MCD in the fullest depth to identify the pros and cons of using MCDs in such ways.

Methods: During problem-based learning, we gave guidelines of MCD for its mechanistic exploration from subcellular processes to clinical features, being laid out in as much detail as possible. To understand the students' attitudes and depth of study using MCDs, we analyzed the results of a questionnaire in an open format about experiencing MCDs and examined the resulting products. Results: Through the responses to questionnaire, we found several favorable outcomes, major of which was deeper insight and comprehensive understanding of disease facilitated by the process of making well-organized diagram. The main disadvantages of these guidelines were the feeling of too much workload and difficulty of finding mechanisms. Students gave suggestions to overcome these problems: cautious reading of comprehensive texts, additional guidance from staff about depth and focus of mechanisms, and cooperative group work. From the analysis of maps, we recognized there should be allowance of diversities in the appearance of maps and many hypothetical connections, which could be related to an insufficient understanding of mechanisms in nature. Conclusion: The more detailed an MCD task is, the better students can become acquainted with deep knowledges. However, this advantage should be balanced by the results that there are many ensuing difficulties for the work and deliberate help plans should be prepared.

Key Words: Case, Mechanisms, Medical education, Problem-based learning

\section{Introduction}

A mechanistic case diagram $(\mathrm{MCD})$ is a graphic representation of the mechanisms of a disease or problems contained within a medical case. MCD can be considered as a special type of concept map in that the former uses rather similar link words between concepts: i.e., linking words used in a MCD are only of similar types such as "causes," "leads to," "results in," and so forth: therefor they are supposed to be hidden from the usual guidelines. Thus the work of making an MCD has been explained as repetitive and consecutive questioning that explores the mechanisms associated with clinical features of disease and laboratory abnormalities and draws connections between the identified concepts,
Received: May 8, 2017 • Revised: July 10, 2017 • Accepted: August 7, 2017 Corresponding Author: Bong Jin Kang (https://orcid.org/0000-0003-4736-797X) Department of Anesthesia and Pain Medicine, Dankook University College of Medicine, 119 Dandae-ro, Dongnam-gu, Cheonan 31116, Korea

Tel: +82.41.550.6819 Fax: +82.41.550.6819 email: anebjkang@hanmail.net
Korean J Med Educ 2017 Sep; 29(3): 153-163.

https://doi.org/10.3946/kjme.2017.61

eISSN: 2005-7288

(C) The Korean Society of Medical Education. All rights reserved. This is an open-access article distributed under the terms of the Creative Commons Attribution Non-Commercial License (http:// creativecommons.org/licenses/by-nc/3.0/), which permits unrestricted non-commercial use, distribution, and reproduction in any medium, provided the original work is properly cited. 
resulting in an integrated map of multiple areas of knowledges. In other words, the work purports to give students fundamental and systematic knowledge for understanding medical problems, beyond rather simple memorization of clinical knowledge, and can thus be described as a method of deep learning [1-3].

MCD work seems to be geared towards modern trends in medical education which emphasize a curriculum that reflects the ever increasing biomedical knowledge that has occurred since the latter half of 20th century [4,5]. However, it has been reported that there exists a scant amount of knowledge that will grant a deep mechanistic understanding for the creation of a well-organized and integrated diagram of the problems in major texts and web-based materials [6]. So, given this situation, our study aims to be a pioneering work that will produce in-depth study materials through the process of making MCDs in the setting of problem-based learning (PBL). Though PBL in medical education intends to develop the skill set necessary to diagnose undifferentiated medical problems at the same time as gaining an understanding of in-depth biomedical knowledge, students can revert to traditional modes of learning if they are not provided with appropriate learning tools [1-3]. The properties of an MCD that facilitate step-by-step mechanistic searches from the etiologic factors to the ultimate clinical features of a disease could invaluably augment in-depth learning.

As far as we know, there have been few studies that explored the ways to utilize MCDs appropriately, at least in the field of medical education. A few studies have reported the use of MCDs during PBL [1,2], but they did not study specific ways of efficiently making MCDs. Regarding the depth of details for making an MCD during PBL, previous authors merely suggested that students make MCDs in as much of a detailed manner as possible [1,2]. On the contrary, Dee et al. [7] suggested a specific set of guidelines including a statement that MCDs for a medical case should be created using at most 25 concepts. However, their guidelines were made according to the rather tight schedule dealing with 44 MCDs over two semesters by each student. In them, the teachers gave preselected lists of concepts below 25 and the individual student made each MCD around the mean time of 15 minutes. So, both in styles of Gurerrero [1], Azer [2], and Dee et al. [7], there have been no controlled studies testing the appropriateness of their methods.

This study reports on the experience of making MCDs during PBL in D Medical School when students followed the guidance to explore disease mechanisms in the fullest depth according the guidelines of articles of Guerrero [1] and Azer [2]. This is qualitative analysis based on the questionnaires and map products. Following this, we intend to find the pros and cons of making MCDs under such a guideline, then establishing more useful guidelines to help students make MCDs during PBL.

\section{Methods}

\section{Participants}

This study involved 45 second-year medical school students at Dankook University, Cheonan, Korea. The mean age of the students was 22 years (standard deviation=1), and they consisted of 34 males (76\%) and 11 females (24\%). The students were divided into six groups, and each group was composed of seven or eight members.

\section{Context and procedure}

The PBL course was held for 1 month at the end of second year of medical school. Students studied one case module for a week. There were no regular lectures except the intermittent special lectures related to the case. Briefly, the four cases were as follows: acute chest pain 
in a middle aged patient (this case was regarded as an exercise for MCD work), seizure in a 26-year-old pregnant patient, low back pain in a 50-year-old female with previous history of breast cancer, fever, and abdominal pain in a 3-year-old child diagnosed as Kawasaki disease.

Each group was supposed to do both of the two types of assignments (PowerPoint [PPT] and MCD) as the final products for one case in a week. Both of the assignments were group work. The PPT assignment was to express students' understanding of differential diagnoses and the management of disease after a diagnosis was reached, and the MCD assignment was meant to illustrate students' understanding of mechanistic explanations of the PBL case. So, MCD was introduced as an aid to in-depth learning focused on the mechanisms of diseases [3].

At the beginning of this study, an instructor gave students an introduction to MCDs. The format of that introductory orientation was based on article of Azer [2], and it covered what an MCD is and how to draw an MCD. In line with the article, we gave students guidelines to perform mechanistic exploration from clinical features to subcellular processes in as much detail as possible when making their MCDs. In other words, investigations into mechanistic processes leading to the various symptoms of each disease (so called pathophysiological mechanisms) and also the mechanistic processes from the etiologic factors to the establishment of diseases (so called pathogenetic mechanisms) were requested in the guidelines [7]. Notably, as part of pathogenetic processes, students were asked to include epidemiological, psychological, and social risk factors in their MCDs and make mechanistic links as much as possible [1,2].

Regarding the actual drawing process, students were given these simple guidelines: (1) Each node corresponding to one concept should only send arrows pointing to the results of the cause or receive arrows from the more causative (higher level in the hierarchical ladder) concepts [1,2]. (2) Important treatments should be expressed at related places in the MCDs using overlaid arrows that are outside of the main stream [1,2]. (3) Each MCD product should be unique and there should be no mindless copying of resource materials. (4) The MCD should be different from mind map that features simple categorization and is composed of etiology, pathogenesis, clinical features, treatments, and so forth, without a detailed investigation of mechanistic processes intertwined over the whole case (https://meducation.net/ resources/31264-Antibiotics-Mindmap). As the first MCD assignment was considered a practice, the researcher did not include data on the first MCD assignment in the analysis for this study and eventually 18 MCDs over 3 weeks were evaluated for this study.

They were encouraged to do group work for MCD after doing an in-depth study on their own learning issues related to MCD. For initial group work, they usually worked using white board and then transcribed the map into open graphical tool, CmapTools (IHMC, Pensacola, USA; http://cmap.ihmc.us/cmaptools/cmaptools-download/) for the MCD assignments. At the end of each PBL session, students gave presentations on their group MCDs. Two MCD committee members evaluated the final MCD products in a subjective manner. They were especially focused on students' comprehension and the completeness of the final $\mathrm{MCD}$, its presentation, the quality of references, integrity of group work, and so on.

\section{Data collection and analysis}

To investigate the students' perspectives on the experience of using MCDs according to our study guidelines, we constructed survey items to assess students' general perceptions of the experience of using an $\mathrm{MCD}$ in such ways during PBL. Three open-ended questions were used to get perspectives concerning the experience of using MCDs and following the instructions of this study: 
(1) What was the hardest aspect of making your group MCDs and how did you overcome it by yourselves? (2) What are the good and bad points of the experience of using MCDs? (3) What are some good strategies that would stimulate each group to make a unique MCD?

The authors gave the questionnaire after the end of fourth week and asked their help to improve this task in the future. They voluntarily participated in the study following the verbal explanation. After collecting the questionnaires, two investigators analyzed the answers independently and categorized the emerging themes. If there were disagreements, they discussed and tried to find solutions. In the case of unclear meaning of the answers, we contacted the students through $\mathrm{e}^{-}$-mail or telephone for verification.

Also the authors observed the MCD products and tried to find the additional pros and cons points of MCD work given our study guidelines. Further during this examination, we tried to get ideas for improving the task.

\section{Results}

\section{Responses to the questionnaire}

Thirty-seven students answered the questionnaire (37/ $45=0.82$ ). Upon observation of the responses to the questionnaire, we could categorize the themes as shown in the Table 1. Students could gain deep understanding of study subject and the benefit of cooperative group work (theme III), but they also felt the need of more guidance and resource materials to relieve their high

Table 1. Analysis of the Responses to the Questionnaire

Categories of the responses

Incidence $(\mathrm{n}=37)$

I. Disadvantages or the most difficult aspects of the task

Difficulty in finding the exact resources exactly explaining the linking mechanisms in question 27

$\begin{array}{ll}\text { Excessive workload and time required for students } & 26\end{array}$

Excessive information collected causing confusion about the essential knowledge 20

An unfaithful attitude of some group members

8

Too deep or too many mechanisms that do not seem to be required in medical education for students 6

II. Coping methods against the disadvantages or difficulties

A cautious reading of comprehensive texts and other references including materials from the related fields of 28 basic sciences

Cooperative team work by all group members

To have a revising step for edition (deletion of overlapped concepts, more orderly array of concepts) or for 20 increasing the visual grasp or readability

Need for guidance from the staff for the direction or focus of search according to the case, considering the 10 vast amount of knowledge

Comments or directions from the staff making it clear in cases of more unknown mechanisms

III. Advantages of using a MCD according to these guidelines

Detailed and deep understanding about the target disease, making it possible to overcome mere memorization 32

Symptoms recognized to be arising from multiple pathways, frequently showing converging or diverging relationships 30

Feel confident regarding the knowledge about diagnosis and treatments 20

To review previously forgotten knowledge of basic sciences 16

Good opportunity for encouraging group members to participate in discussion owing to their own efforts for 9 studying the mechanisms and the resulting confidence in their assigned parts

A new and interesting way of study 
Table 1. (Continued)

$$
\text { Categories of the responses }
$$

Incidence $(\mathrm{n}=37)$

IV. Ideas to create their own MCDs in a more thoughtful and effortful way; or ideas to discriminate the products created by a simple copying attitude

To evaluate whether a certain MCD product excessively uses the same word or sentence in the textbook

or draws overly simplistic linear diagrams without enough ramifications

To perform more search until obtaining a more adequate and understandable explanation

To provide questions to the group members during the presentation time, for example, about whether they understood the map contents in a deep sense

To clearly write the names of members in the annotation part of the map

To report the names of unfaithful members to teachers through an individual route

7

MCD: Mechanistic case diagram.

Fig. 1. Parts of MCD for a Case Describing 50-Year-Old Female Patient with a Chief Complaint of Severe Low Back Pain

(A)
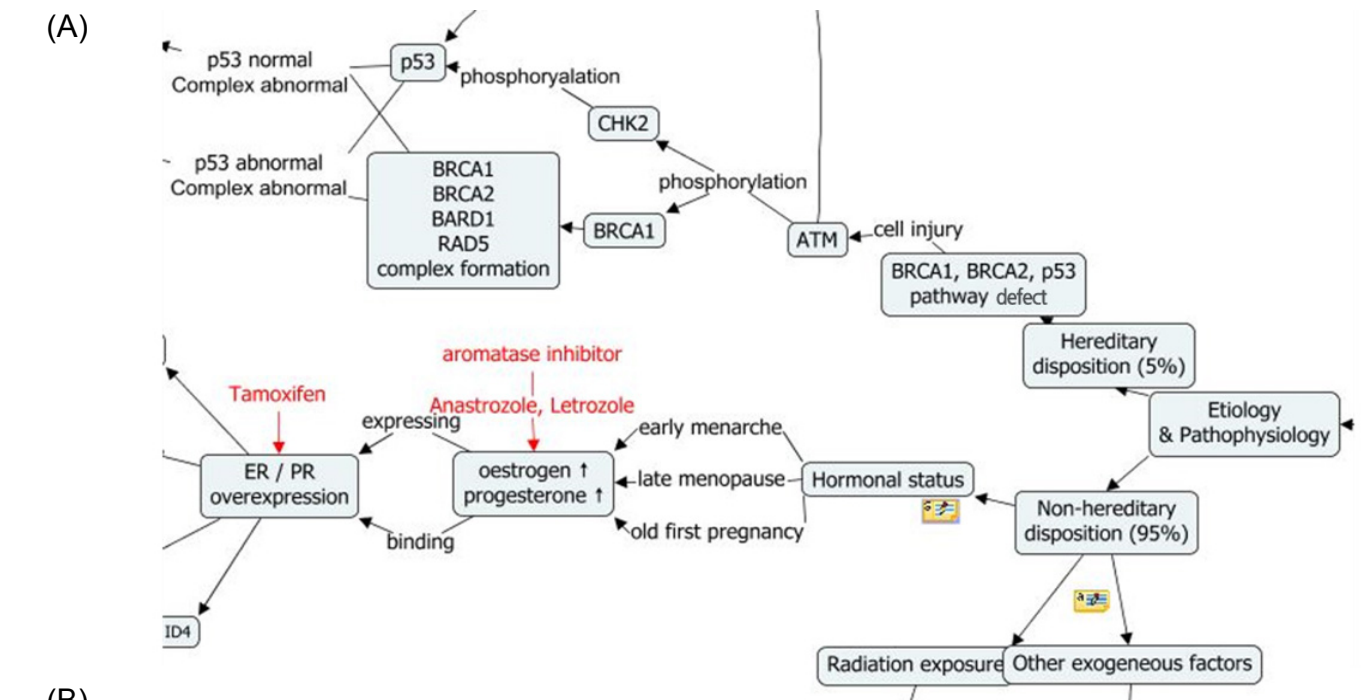

disposition (5\%)

(B)

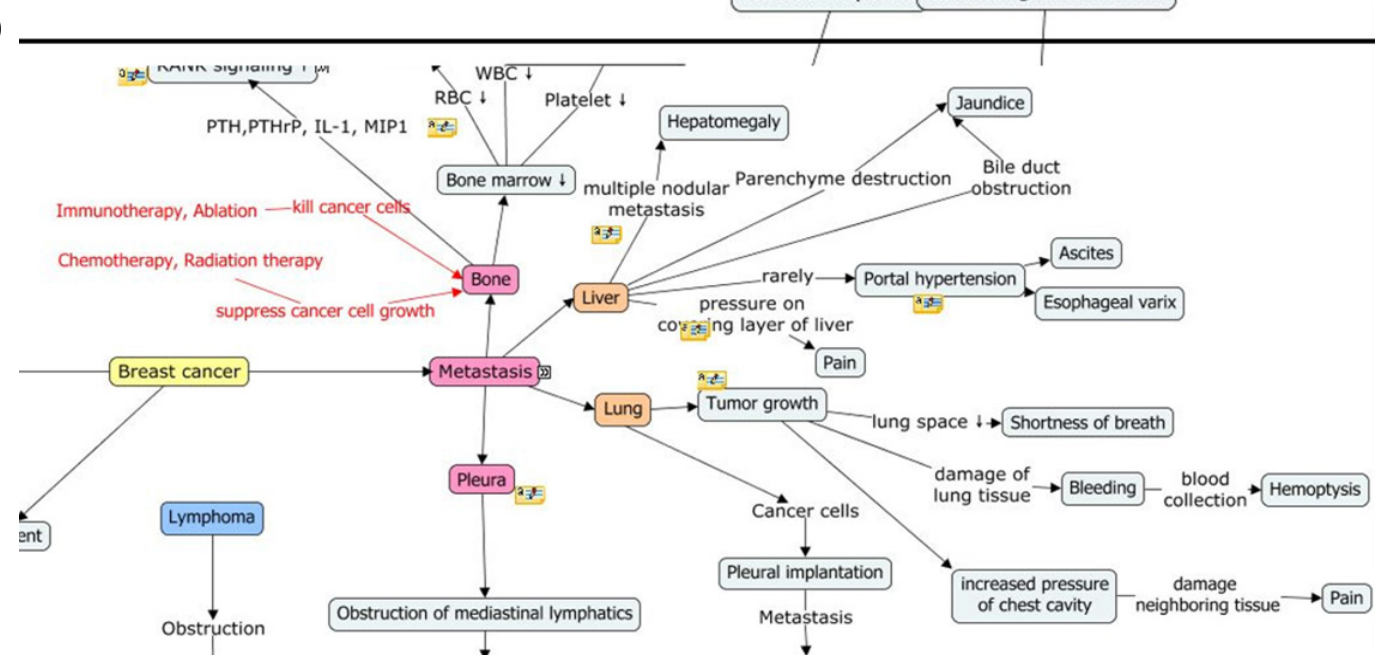

(A) Upper part is from etiopathogenesis and (B) lower part is from some portion of clinical features. She had a history of an operation for breast cancer. At this time, she was showing decreased mentality and multiple metastases in the bones, lungs, and liver as well as various abnormalities on blood tests according to further evaluation. This mechanistic case diagram (MCD) shows the pathogenetic processes and the mechanistic processes that led to diverse clinical manifestations in one figure. It also shows the sites of action of various treatments in red letters. The original file of this MCD product is available in CMap format after downloading of CmapTools or JPG format. 
workload (theme I). The authors could derive some ideas to overcome these difficulties regarding the MCD task from students (themes II and IV).

\section{MCD products}

By observing the MCD products, we found several characteristic patterns. First, regarding general appearance of the MCDs, there emerged a pattern which was quite different than previously introduced as the standard form. The original pattern showed all concepts, from the etiologic factors to the establishment of a disease (pathogenetic processes), then the clinical features and laboratory results (pathophysiological processes) in one picture (Fig. 1). The new pattern which gave significant attention to the visual acceptability of map, edited the comprehensive and complicated pattern into a packaged form composed of key sections such as pathogenetic mechanisms, risk factors, and clinical

Fig. 2. Packed and Unpacked Form of a Different Format of MCD Which Was Made for the Same Case in Fig. 1

(A) yellow box: patient's symptoms

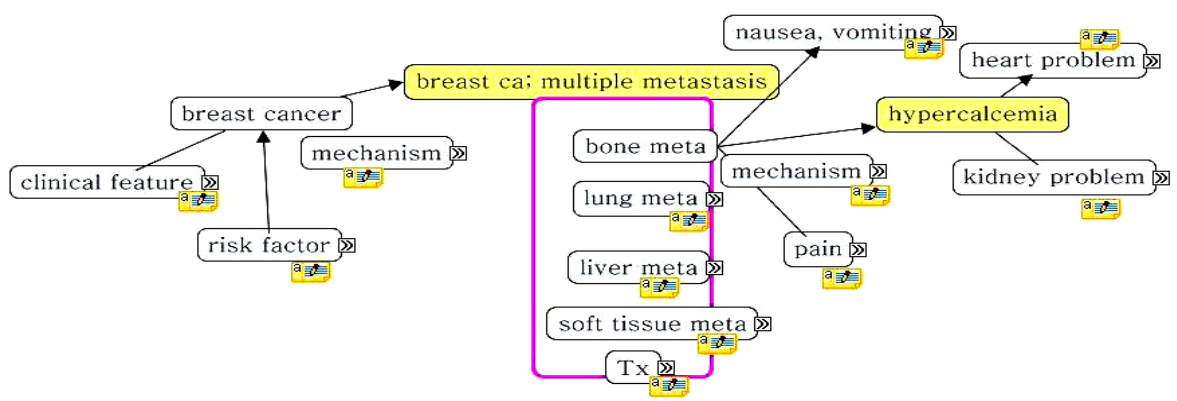

(B)

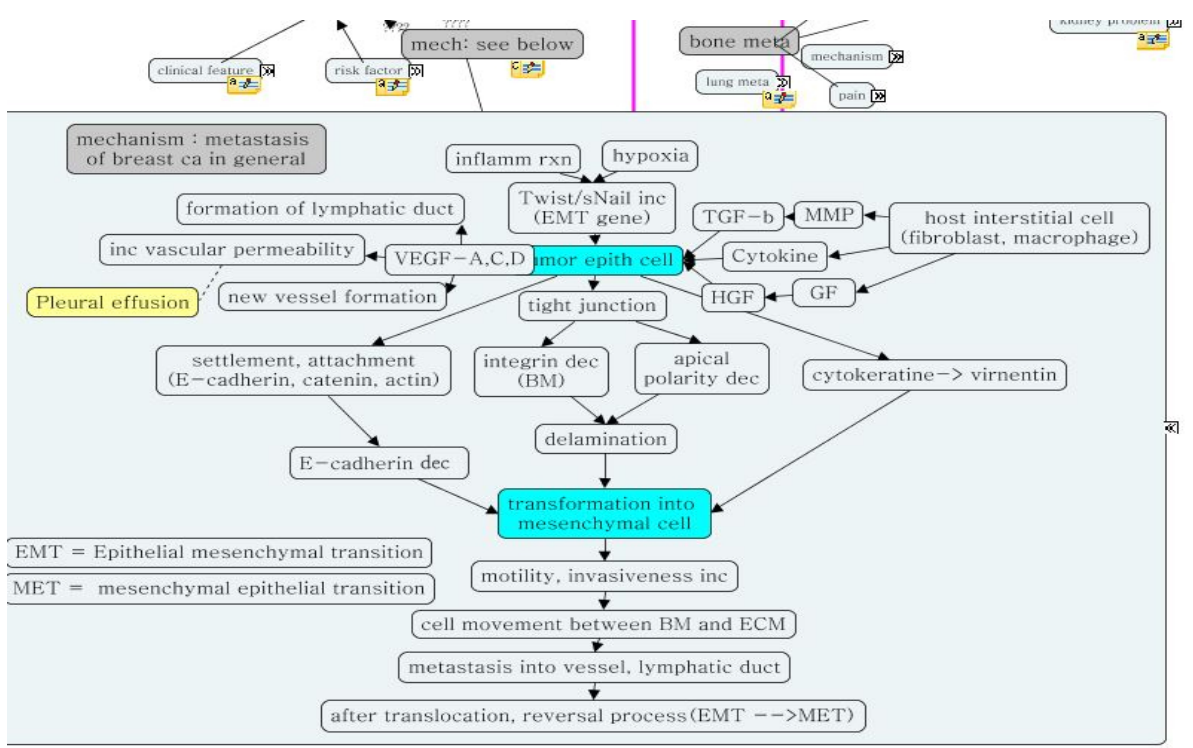

(A) Packed form. (B) Partially unpacked form of (A). Students, considering the complexities of following the style of Fig. 1, drew many boxes of subtopics each including a vast amount of underlying details within each. This pattern does not seem to disobey the basic principles of making an mechanistic case diagram (MCD), that is, connecting all the details along the lines of mechanistic explanations (A, B). If one clicks the ">>" icons in (A), the detailed mechanisms appear, as in (B). (B) shows some concealed parts of clinical features concerning the pathogenesis of breast cancer and bone metastasis. We can see treatment points in red letters in the JPG format of figure (B). The original file of the MCD products is available in (A) CMap format after downloading of CmapTools or JPG format; (B) CMap format after downloading of CmapTools or JPG format. Tx: Treatment, EMT: Epithelial mesenchymal transition, TGF- $\beta$ : Transforming growth factor $\beta$, MMP: Matrix metalloproteinase, VEGF: Vascular endothelial growth factor, HGF: Hepatocyte growth factor, GF: Growth factor, BM: Basement membrane, ECM: Extracellular matrix, MET: Mesenchymal epithelial transition. 
features: each section gave cautions to contain detailed information linked with mechanistic processes, thereby differentiating the MCDs from simple categorization
(Fig. 2A, packed form; 2B, partially unfolded form). This pattern emerged out from the purpose to get rid of too much visual information in the initial map and show

\section{Fig. 3. Parts of MCD for a Case Describing a 3-Year-Old Child with Chief Complaints of Fever and Abdominal Pain}

(A)

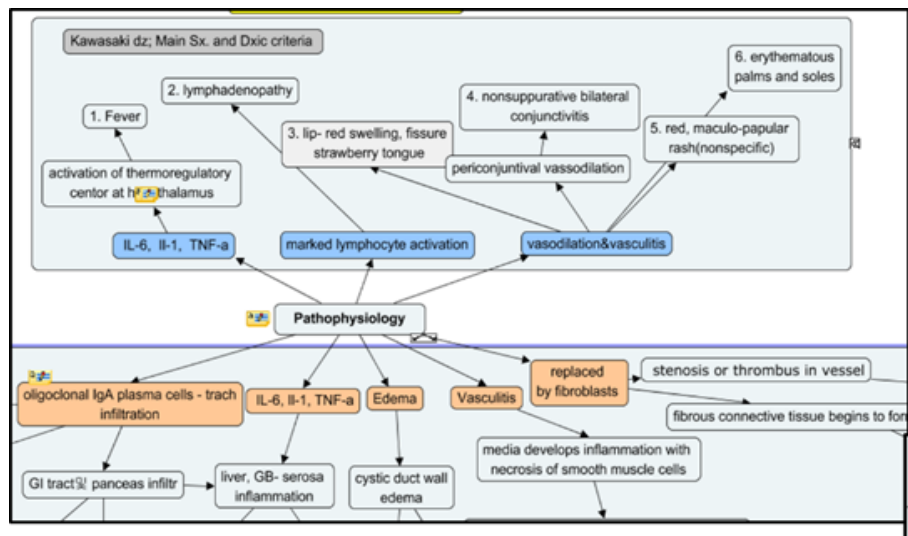

(B)

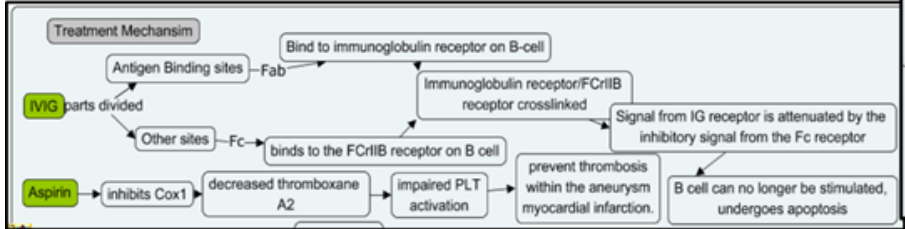

(C)

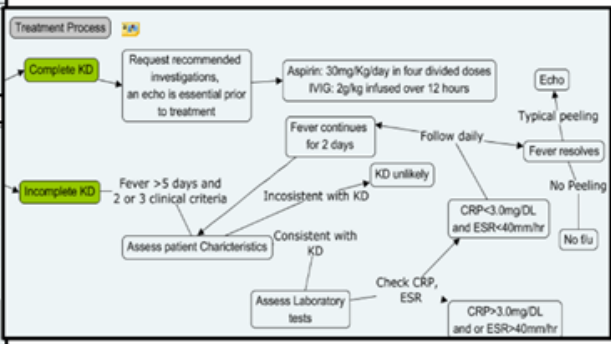

(A) Captured from a portion of clinical features also with a box showing diagnostic criteria. (B) Captured from a portion of additional box showing mechanisms of all treatments. (C) Captured from a portion of additional box showing treatment algorithms according to the types of Kawasaki disease (KD). His condition was diagnosed as KD, a type of autoimmune disease, based on the findings of a truncal rash, hematologic changes (leukocytosis, high C-reactive protein [CRP], elevated liver enzymes, etc.) and abnormal echocardiography. This mechanistic case diagram (MCD), peculiarly, shows the box containing diagnostic criteria in the middle and other various boxes related to the diverse sub-topics of treatments (treatments in general, treatments for complete or incomplete forms of KD) on the right side. The original file of the MCD products is available in CMap format after downloading of CmapTools or JPG format. Sx: Symptom, IL: Interleukin, TNF-a: Tumor necrosis factor a, GI: Gastrointestinal, GB: Gallbladder, IVIG: Intravenous immunoglobulin, PLT: Platelet, DL: Per Deciliter, ESR: Erythrocyte sedimentation rate.

Fig. 4. Incomplete or Well Organized Form of Drawing for a Case of a 26-Year-Old Pregnant Patient with a Chief Complaint of an Abrupt Seizure

(A)

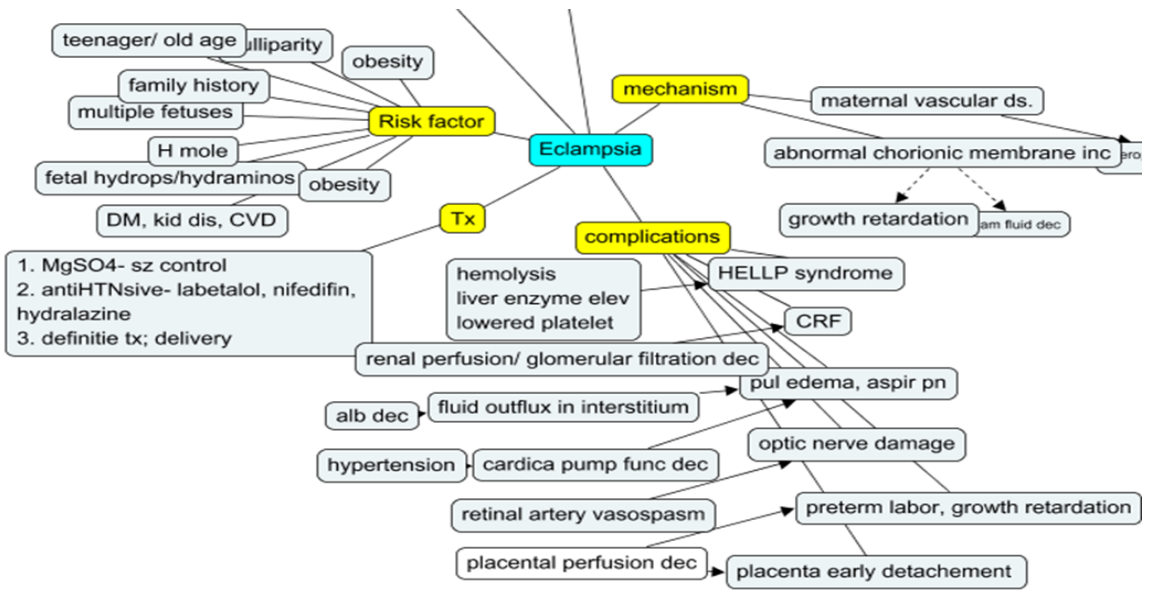

(Continued to the next page) 
Fig. 4. (Continued)

(B)

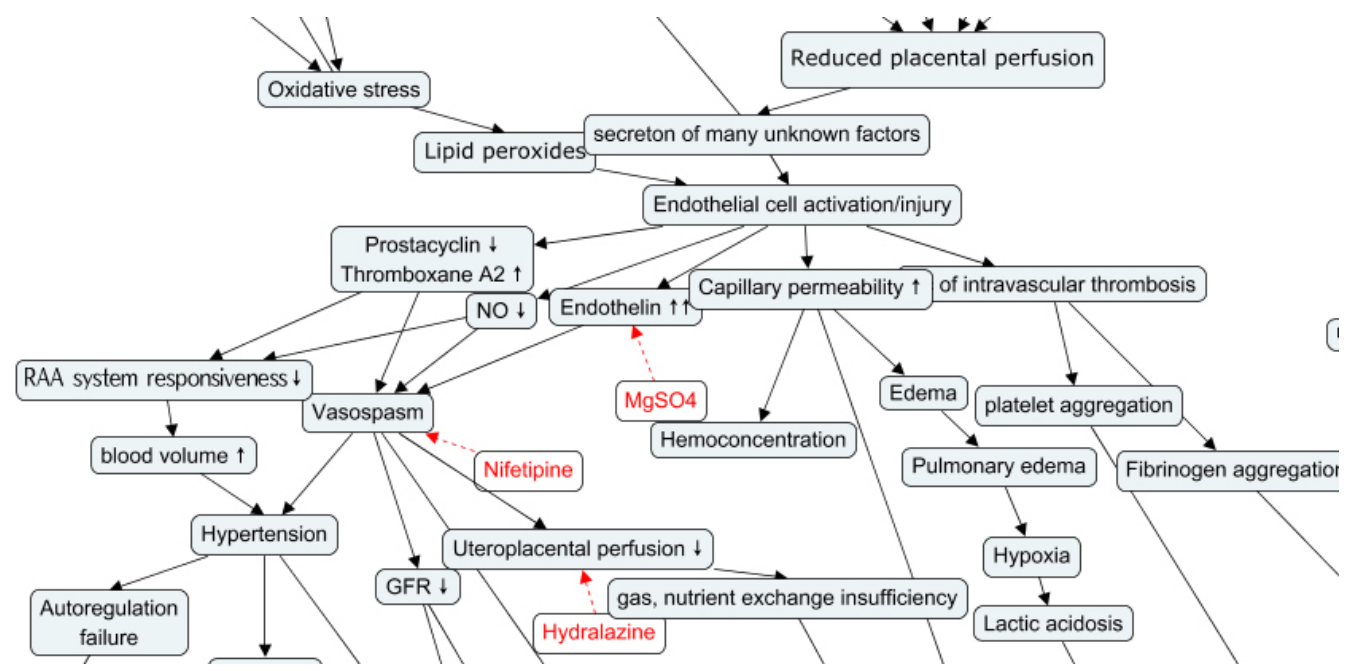

(A) Incomplete or abbreviated form of drawing for a case of a 26-year-old pregnant patient with a chief complaint of an abrupt seizure. (B) More organized form mechanistic case diagram (MCD) compared with (A) which came from another group. She was then diagnosed with eclampsia. (A) shows a rather abbreviated drawing for the patient's clinical features, which is contrasted with the other parts of the same diagram (for example, the pathogenesis part). This result probably reflects incomplete cooperation between group members or the nature of the heavy workload associated with creation of the MCD. (B) Which is the product of another group shows more and well organized information over the whole map. The original file of this MCD product is available in (A) CMap format after downloading of CmapTools or JPG format; (B) CMap format after downloading of CmapTools or JPG format. DM: Diabetes mellitus, CVD: Cardiovascular disease, Tx: Treatment, HELLP: Hemolysis, Elevated Liver enzyme, Low platelet count, CRF: Chronic renal failure, RAA: Renin, angiotensin, aldosterone, GFR: Glomerular filtration rate.

gradual mechanistic concepts in a flexible manner. So, the originally introduced pattern was shown in 11 out of 18 and new pattern in seven out of 18.

Second, we frequently observed new map components in addition to the required basic components of MCD; they appeared as boxed summaries under the small topics like diagnostic criteria or all treatments arranged as algorithmic form (Fig. 3). Students wanted to have an opportunity to express important points in the form of boxes, though they seem to be redundant.

Third, some groups showed a failure in detailing parts of the MCD, which was presumably caused by insincere attitudes held by some group members (Fig. 4A in comparison with Fig. 4B). Also the problem of too much workload expressed by many students could explain this in part.

Fourth, there were general deficiencies in illustrating the mechanistic processes related to risk factors except the relatively well known area related to molecular pathogenesis.

\section{Discussion}

The authors interpreted the guidelines in key references [1,2] as in this way: be detailed as much as possible and connect all the minute details from risk factors to clinical features. Those guidelines also encouraged multiple converging or diverging relationships between concepts.

Upon the observations of our study, the expected benefits were frequently reported: Students found upon their own extensive searches that symptoms could be manifested through the processes of diverse body systems and also interconnected with many other concepts. This kind of mechanistic understanding facilitated deep learning which was never experienced under the traditional method of study based on didactic lecture 
or even the study for PPT assignment work during PBL. Furthermore a large amount of work pushed the students to do organized group work such as appropriate division of learning issues and integration at a later time.

However, they simultaneously felt a lot of difficulties or shortcomings of the MCD task following our given guidelines. Many students complained about the need of too much work and the difficulty to discriminate out the essential knowledge. Also they wanted more guidance and resources especially in the cases with many difficult-to-find or probably unknown mechanisms. Among MCD products evaluated as having poor quality, some showed parts of the diagram in an incompletely drawn state sharing features of mind map (Fig. 4A, "clinical features" part). This seems to reflect the difficulties of students regarding their workload, shortage of time and the need of deliberate guidance of the committee. Regarding the work related to risk factors within the MCDs, many groups ended up by listing all the factors in the same concept box and then linked it roughly to the concept box of disease name even though we requested to make mechanistic linkages for each risk factor (for example, Fig. 4A, "risk factors" part). This reflects the scarcity of those mechanisms in many textbooks. Also, it indicates the area related to the mechanisms of risk factors are relatively more unclear and still in stage for searching or formulating more mechanisms [8-11].

We could not expect those main difficulties related to too much workload at the time of study. But after seeing the responses to the questionnaires and some poorly qualified MCD products, now we have new plans to be applied for later applications of MCDs. (1) People composed of case expert and members of the operating committee for MCDs should meet earlier time enough, discuss the purpose of MCDs and make MCDs in advance. Further at that time, we should discuss about the required depth of the mechanisms, the area of more focus and ways of expression in the areas of more uncertainty regarding mechanisms, among others. (2) During the above meeting, we should recognize many unknown or uncertain mechanistic details with regards to the object case. Then we should somehow discriminate the linkages of hypothetical nature in many points and also accept the fact that it can be premature for any mechanistic formulation to cover up minute details [8-11]. (3) Considering students' unfamiliarity about MCD works, we should prepare good examples of MCD. Also in order to decrease the burden, it will be helpful to prepare a paper describing various tips for doing through the work in general and for each specific case (for example, "List up the keywords including from the important symptoms to the underlying concepts of basic sciences like inflammation, immune response, neoplastic change, etc.," "Plan a balanced mechanism conveying the big picture and fine details," "Consider the factors like the exposure to infectious agents or environmental factors and their possible mechanisms in this case."). (4) Remind the importance of efficient group work and prepare the measures to defeat the discouraging attitudes of some group members (for example, "Give checking up questions to random group members," "Introduce the names of insincere members through the individual route."). (5) Considering relatively deficient amount in resources for risk factors with their mechanistic explanations, we should prepare in advance and introduce them to students.

We found additional benefits and lessons for later use through the analysis of MCD products. For example, we could see modified form which was developed from the intention that tries to improve the complex nature of MCDs (Fig. 2). This type of map was influential to the other groups resulting in six more products of its similar kinds. Williams et al. [12] also gave considerations to the 
problem of complexity and suggested a map which allows each student to navigate the content and level of detail. They called it "adaptive concept map." Similarly, we think it is a good advice to suggest that students have an idea to edit or revise their maps for increasing visual acceptability.

In some MCDs, additional components upon the required basic components - that is to say all the pathogenetic and pathophysiologic concepts-appeared. They were boxed summaries showing diagnostic criteria or treatments, sometimes differentiating into diagnostic subsets (for example, incomplete or complete Kawasaki disease) or situationally specific treatments (for example, treatments for incomplete or complete Kawasaki disease). Further, treatments could be shown as an algorithmic form facilitating the understanding of specific situations regarding treatments (Fig. 3). These summarizing box contents seem to be redundant over the basic contents of MCDs, but we think they can be useful learning activity by reutilizing or reanalyzing the concepts already expressed in the basic MCD and helping to grasp more practical forms of knowledge in clinical field.

Furthermore, from the literature survey, we could see other potential values of MCDs for several issues related to medical education. First, many authors stress the importance of understanding the complex natures of scientific problems including medical field, suggesting the ever existing need of comprehensive studies of disease mechanisms and the need of study tool like MCD [13-15]. Second, MCD can also be a useful educational tool which can goes with one recent trend stressing holistic systems biology applied to human disease. This approach is emerging as the alternative to overcome the shortcomings of traditional medicine giving limited explanation of diseases and their treatments [16,17]. Third, MCD can also be a useful educational tool in these days stressing the importance of integration between basic science and clinical knowledges [5,18,19]. Regarding this point, specifically if we know how to control the depth of content details required for the educational purposes, it could be a more valuable tool to be used flexibly according to the curricular purposes. Fourth, considering the constant development of new biomarkers for early diagnosis, prognostic evaluation or new treatment, MCD can be a useful guide of study to the expanding field of knowledge.

Among the limitations of this study, it should be noted that the main use of brief questionnaire could only elicit rather superficial responses from students. Thus the facts that we did not directly observe the process of creating a MCD or the actual group work limits our understanding of the pros and cons of this MCD work. If we add planned interviews or direct observations of students' work at a later time, it will help us get more understanding regarding this MCD task.

In conclusion, the more detailed an MCD task is, the more students will grasp in-depth knowledge of a disease. However, this policy inevitably involves giving students a high workload, suggesting that many deliberate learning aids or tips are prepared in advance.

\section{ORCID:}

Minjeong Kim: https://orcid.org/0000-0002-5117-7071; Bong Jin Kang: https://orcid.org/0000-0003-4736-797X Acknowledgements: None.

Funding: None.

Conflicts of interest: None.

Author contributions: Conception or design of the work: BJK and MK. Data collection: BJK. Data analysis and interpretation: BJK and MK. Drafting the article, critical revision of the article, and final approval of the version to be published: BJK and MK. 


\section{References}

1. Guerrero AP. Mechanistic case diagramming: a tool for problem-based learning. Acad Med. 2001;76(4):385-389.

2. Azer SA. Facilitation of students' discussion in problembased learning tutorials to create mechanisms: the use of five key questions. Ann Acad Med Singapore. 2005; 34(8):492-498.

3. Kang BJ. Experience with using multiple types of visual educational tools during problem-based learning. Korean J Med Educ. 2012;24(2):127-139.

4. Tomorrow's doctors-outcomes and standards for undergraduate medical education. General Medical Council Web Site. http://www.gmc-uk.org/publications/undergraduate_ education_publications.asp. Accessed May 1, 2017.

5. Finnerty EP, Chauvin S, Bonaminio G, Andrews M, Carroll RG, Pangaro LN. Flexner revisited: the role and value of the basic sciences in medical education. Acad Med. 2010;85(2):349-355.

6. Azer SA. Mechanisms in cardiovascular diseases: how useful are medical textbooks, eMedicine, and YouTube? Adv Physiol Educ. 2014;38(2):124-134.

7. Dee FR, Haugen TH, Kreiter CD. New web-based applications for mechanistic case diagramming. Med Educ Online. 2014;19(1):24708.

8. Arbab-Zadeh A, Nakano M, Virmani R, Fuster V. Acute coronary events. Circulation. 2012;125(9):1147-1156.

9. Inouye SK, Studenski S, Tinetti ME, Kuchel GA. Geriatric syndromes: clinical, research, and policy implications of a core geriatric concept. J Am Geriatr Soc. 2007;55(5):780-791.

10. Gourley M, Miller FW. Mechanisms of disease: environmental factors in the pathogenesis of rheumatic disease. Nat Clin Pract Rheumatol. 2007;3(3):172-180.

11. Wilson IB, Cleary PD. Linking clinical variables with health-related quality of life: a conceptual model of patient outcomes. JAMA. 1995;273(1):59-65.

12. Williams CB, Moore JP, Johri A, Pierce RS, North C. Advancing personalized engineering learning via an adaptive concept map. Paper presented at: 2012 ASEE Annual Conference \& Exposition; June 10-13, 2012; San Antonio, USA. https://peer.asee.org/20897. Accessed May $1,2017$.

13. Spiro RJ. Multiple analogies for complex concepts: antidote for analogy-induced misconception in advanced knowledge acquisition: the Reading Research and Education Center report. https://eric.ed.gov/?id=ED301873. Published September 1988. Accessed May 1, 2017.

14. Feltovich PJ, Spiro RJ, Coulson R. The nature of conceptual understanding in biomedicine: the deep structure of complex ideas and the development of misconceptions. In: Evans DA, Patel VL, eds. Cognitive science in medicine: biomedical modelling. Cambridge, USA: MIT Press; 1989.

15. Perkins DN, Grotzer TA. Models and moves: focusing on dimensions of causal complexity to achieve deeper scientific understanding: report of the American Educational Research Association Annual Conference. https://eric.ed.gov/?id=ED441698. Published April 2000. Accessed May 1, 2017.

16. Loscalzo J, Barabasi AL. Systems biology and the future of medicine. Wiley Interdiscip Rev Syst Biol Med. $2011 ; 3(6): 619-627$.

17. Margineanu DG. Systems biology impact on antiepileptic drug discovery. Epilepsy Res. 2012;98(2-3):104-115.

18. Eisenbarth S, Tilling T, Lueerss E, et al. Exploring the value and role of integrated supportive science courses in the reformed medical curriculum iMED: a mixed methods study. BMC Med Educ. 2016;16:132.

19. Azzalis LA, Giavarotti L, Sato SN, Barros NM, Junqueira $\mathrm{VB}$, Fonseca FL. Integration of basic sciences in health's courses. Biochem Mol Biol Educ. 2012;40(3):204-208. 